\title{
On the fate of extremely low metallicity stars
}

\author{
P. Gil-Pons*, J.L. Gutiérrez*, E. García-Berro* and L. Althaus ${ }^{\dagger}$ \\ * Departament de Física Aplicada, Universitat Politècnica de Catalunya, Av. Canal Olímpic s/n, \\ 08860 Castelldefels, Spain \\ ${ }^{\dagger}$ Facultad de Ciencias Astronómicas y Geofísicas, Universidad Nacional de La Plata, Paseo del \\ Bosque s/n, B1900FWA, La Plata, Argentina
}

\begin{abstract}
We have computed and analyzed the evolution of intermediate-mass stars of metallicity $Z=10^{-5}$ from the main sequence until the early stages of the TP-(S)AGB phase. In order to check the influence of mixing we have performed our calculations using two different evolutionary codes, EVOLVE — which does not include diffusion but allows for overshooting - and LPCODE, which includes diffusion and overshooting "à-la-Herwig". Important differences appear during the TPAGB phase, as the calculations done with EVOLVE lead to an almost negligible third dredge-up, whereas the sequences computed with LPCODE show important third dredge-up and, therefore, significant enrichment in metals of the envelope. This fact has consequences on the strength of the winds during the TP-AGB phase and, ultimately, on the final fate of the considered stars. Also, the models computed with LPCODE show strong double flashes produced by the ingestion of protons on the helium burning shell.
\end{abstract}

Keywords: Stellar structure, Abundances, Population III stars, Supernovae

PACS: $97.10 . \mathrm{Cv}, 97.10 . \mathrm{Tk}, 97.20 . \mathrm{Wt}, 97.60 . \mathrm{Bw}$

\section{INTRODUCTION}

Primordial and extremely low metallicity stars are drawing increasing attention, not only because we have now a harvest of new observations of more metal poor environments $[1,2]$, but also because of the recent progresses achieved in the theoretical calculations. Recent studies [3, 4] have found peculiar flashes that ensue during the evolution of extremely metal poor stars, which could dramatically affect - and be affected by the mixing mechanisms that must be taken into account. We might be facing a problem not to be accounted for either by the standard mixing length recipes nor by diffusion. Instead, inputs from multidimensional hydrodynamic simulations might be required $[4,5]$. Moreover, recent calculations of the evolution of intermediate-mass primordial stars $[6,7,8]$, point out to the possibility that these stars might end their lives as SNeI1/2 [9], since the initial lack of metals of the envelope and the inefficiency of the dredge-up process favour very weak winds, that are unable to eject the envelope before the core reaches the Chandrasekhar mass.

In this work we explore the effects of the initial metallicity on the late stages and final fate of intermediate-mass stars of extremely low metallicity $\left(Z=10^{-5}\right)$, and we discuss whether the results obtained for their counterparts of primordial composition hold for these objects. We target as well the consequences of different mixing prescriptions. Finally, we also assess the impact of the numerical schemes adopted in the evolutionary codes, by computing evolutionary sequences with two different codes. In section 2 we describe the codes and we outline the evolution during the main central burning stages. 


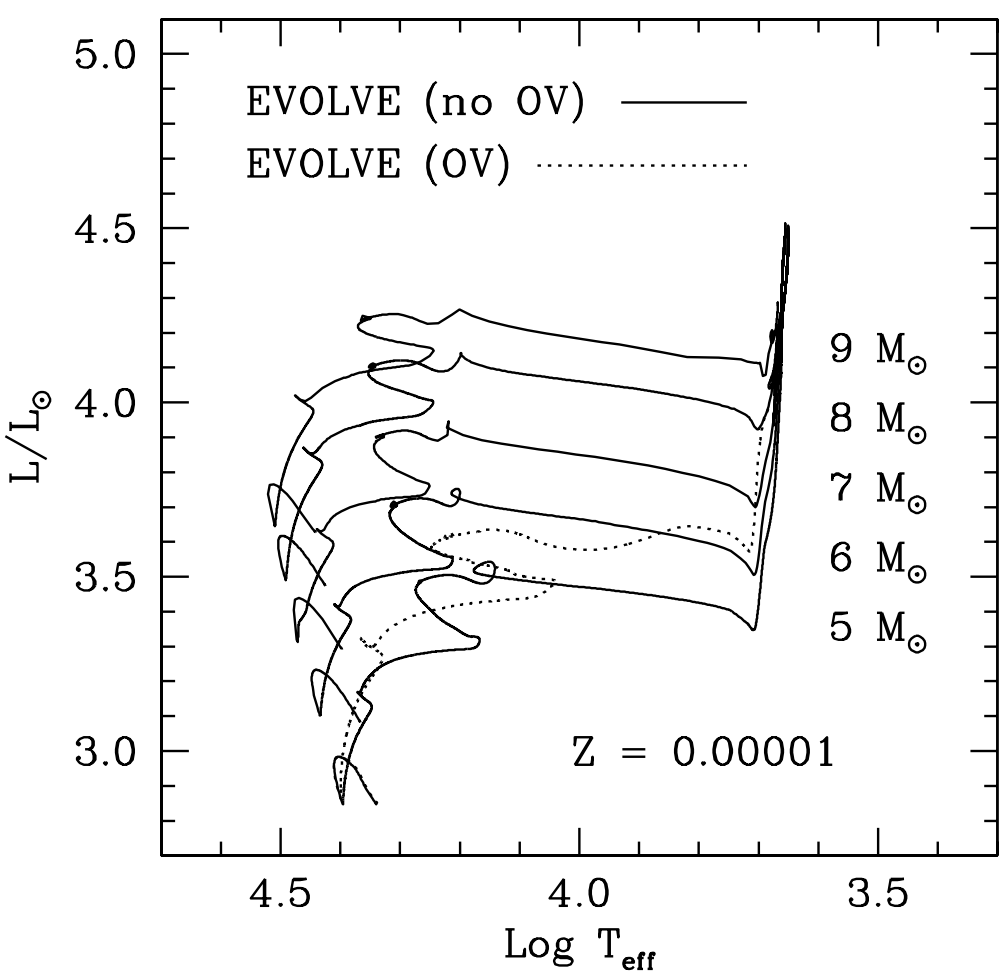

FIGURE 1. Evolution in the Hertzsprung-Russell diagram of our $Z=10^{-5}$ models computed with EVOLVE.

In section 3 we describe the early stages of the TP-AGB phase and focus on the problem of their final fate, whereas in the last section we summarize our findings and we draw the main conclusions of our work.

\section{INPUTS, EVOLUTIONARY CODES AND SEQUENCES}

In order to check how the mixing prescriptions affect the evolution of extremely low metallicity intermediate-mass stars, we have computed a series of sequences using two different codes: EVOLVE and LPCODE. The evolutionary code EVOLVE follows the Hand He-burning phases and has been updated to include carbon burning [10] and the new OPAL opacities [11], but does not include element diffusion. Recently, it has been used to analyze intermediate-mass primordial stars and how overshooting can affect their main properties $[11,7]$. The treatment of overshooting has been implemented following Refs. [12] and [13]. To be precise, convection sets in when $\nabla_{\text {rad }}>\nabla_{\text {ad }}-\delta$, where $\delta$ depends on the ratio $P_{\text {rad }} / P_{\text {gas }}$ and on a free parameter that has been calibrated in order to fit observations. Using EVOLVE without overshooting we have computed the evolution of $Z=10^{-5}$ stars of initial masses between 5 and $9 M_{\odot}$. For the sake of comparison, we have also computed the evolution of a $5 M_{\odot}$ model star with overshooting. The tracks in the Hertzsprung-Russell diagram of these model sequences are shown in figure 1. 

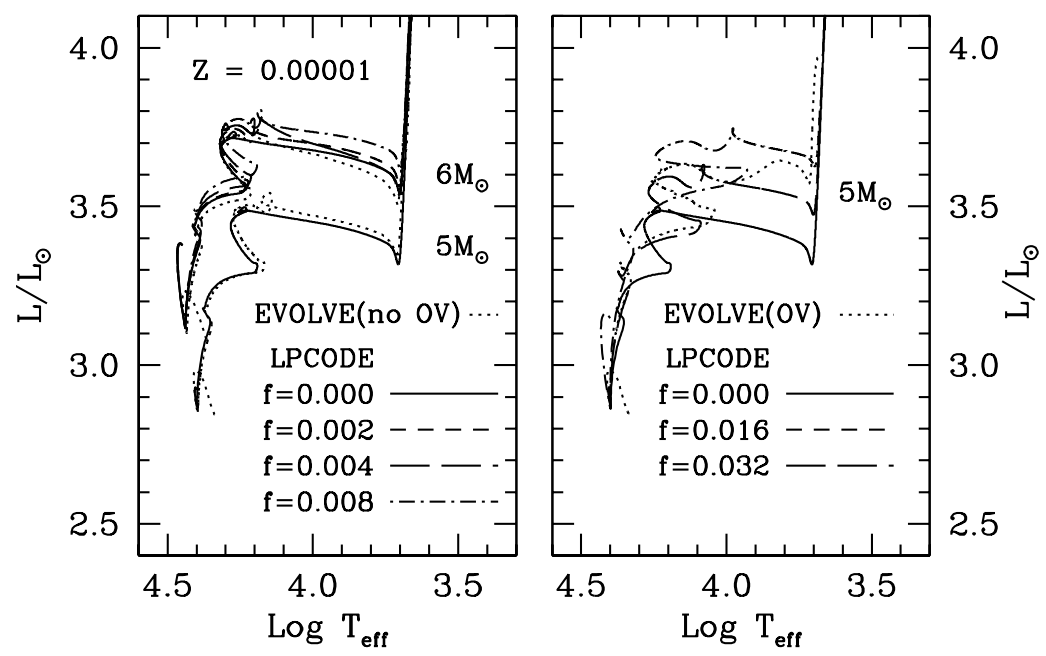

FIGURE 2. Evolution in the Hertzsprung-Russell diagram of our $Z=10^{-5}$ models computed using LPCODE and several values of $f$

LPCODE [14] includes diffusion and the treatment of overshooting follows the prescription of Ref. [15]. That is, convective mixing is extended beyond the frontier of stability according to the exponentially decaying law:

$$
D_{\mathrm{OV}}=D_{0} \exp \left(\frac{-2 z}{H_{v}}\right)
$$

where the parameters $D_{0}=v_{0} H_{P}, z=\left|r_{\text {edge }}-r\right|$ and $H_{v}=f H_{P}$. The parameter $f$ adopts values between 0 and 0.032 , although the value recommended lately is 0.008 [16]. Larger values of $f$ imply a more extended mixing. Unlike EVOLVE, LPCODE does not allow to compute the carbon burning phase.

We have analyzed the evolution of $5 M_{\odot}$ and $6 M_{\odot}$ stars, with composition $X=0.755$, $Y=0.245$ and $Z=10^{-5}$, and with different values of $f$, ranging from 0.0 to 0.032 . The left panel of figure 2 shows the tracks in the Hertzsprung-Russell diagram of the models computed with LPCODE and $f$ between 0.0 and 0.008 . The right panel of the same figure shows the evolution in this diagram of the $5 M_{\odot}$ model computed with EVOLVE - with and without overshooting - and with LPCODE and values for $f$ of 0.016 and 0.032 . As can be seen in the figure, the amount of extra mixing due to overshooting obtained using EVOLVE corresponds to a fairly large value of $f$, intermediate between 0.016 and 0.032 . On the other hand, the sequences computed with no overshooting show a fair agreement.

\section{Evolution during the main central burning stages}

Opposite to what happens in the case of primordial stars, stars with a metallicity of $Z=10^{-5}$ develop core hydrogen burning (CHB) through the CNO cycle, as the initial amount of metals is more than enough to allow the onset of these reactions. However, 
TABLE 1. Masses of the $\mathrm{CO}$ cores and central abundances of carbon and oxygen for the $6 M_{\odot}$ model stars computed with EVOLVE and no overshooting and with LPCODE with several different values of $f$.

\begin{tabular}{ccccc}
\hline Code & $f$ & $M_{\mathrm{CO}}$ & $X_{\mathrm{c}}\left({ }^{12} \mathbf{C}\right)$ & $X_{\mathrm{c}}\left({ }^{16} \mathbf{O}\right)$ \\
\hline EVOLVE & - & 0.96 & 0.31 & 0.69 \\
LPCODE & 0.000 & 0.85 & 0.41 & 0.59 \\
LPCODE & 0.002 & 0.87 & 0.32 & 0.68 \\
LPCODE & 0.004 & 0.88 & 0.26 & 0.74 \\
LPCODE & 0.008 & 0.92 & 0.36 & 0.64 \\
LPCODE & 0.016 & 0.98 & 0.34 & 0.66 \\
\hline
\end{tabular}

the tracks of primordial and $Z=10^{-5}$ stars show some aspects in common. For instance, they do not climb the red giant branch during hydrogen shell burning but instead, they do it for the first time during the helium shell burning phase. The reason for such behavior is that the cores are so hot and compact at the end of the CHB phase that the $3 \alpha$ reaction sets in before the hydrogen-burning shell is able to induce the expansion and cooling of the stellar envelope. Therefore both $\mathrm{CHB}$ and core helium-core burning $(\mathrm{CHeB})$ take place at the left hand side of the Hertzsprung-Russell diagram. This general behavior is independent of the code used and of the value adopted for $f$.

As for the evolutionary timescales, the $Z=10^{-5}$ models computed with EVOLVE yield values longer than those corresponding to primordial stars obtained with the same code [7]. Also, the masses of the degenerate cores are larger and the central abundances of carbon (oxygen) are smaller (larger) than those of the corresponding primordial cases. When the sizes of the cores obtained with the two codes are compared, those resulting from the LPCODE sequences and $f=0$ are about $12 \%$ less massive than the cores computed with EVOLVE - see Table 1 . As $f$ increases and reaches values ranging from 0.016 to 0.032 , the $\mathrm{CO}$ core masses become similar for both codes. Finally, with regard to the ratio $X(\mathrm{C}) / X(\mathrm{O})$, the $5 M_{\odot}$ sequence computed with EVOLVE also yields a value similar to that of the corresponding LPCODE model and $f=0.016$.

Carbon burning in our $Z=10^{-5}$ models develops in conditions of partial degeneracy and, therefore, through a series of flashes in which the carbon luminosity, $L_{C}$, can reach values as large as $\sim 10^{8} L_{\odot}$. This large release of energy causes a fast increase in the temperature gradient and the development of associated convective shells. The expansion and cooling of the layers just above causes the temporary switch-off of the helium-burning shell (HeBS), that recovers as soon as the carbon flash finishes.

Figure 3 shows the temporal evolution of the carbon $\left(L_{\mathrm{C}}\right)$ and helium $\left(L_{\mathrm{He}}\right)$ luminosities (upper panel) and the convective zones (lower panel) during the carbon-burning phase. As can be seen, the burning flame reaches the centre of the star after the second flash. The most interesting feature we have found in this case is related to the evolution of convection during the last phases of carbon burning: convection advances inwards in the stellar envelope after the onset of the HeBS and stops its advance when carbon burning begins at a mass point of $0.19 M_{\odot}$. But when the last convective zone associated to carbon burning is about to disappear and $L_{\mathrm{C}}<10^{4} L_{\odot}$, the base of the convective envelope 


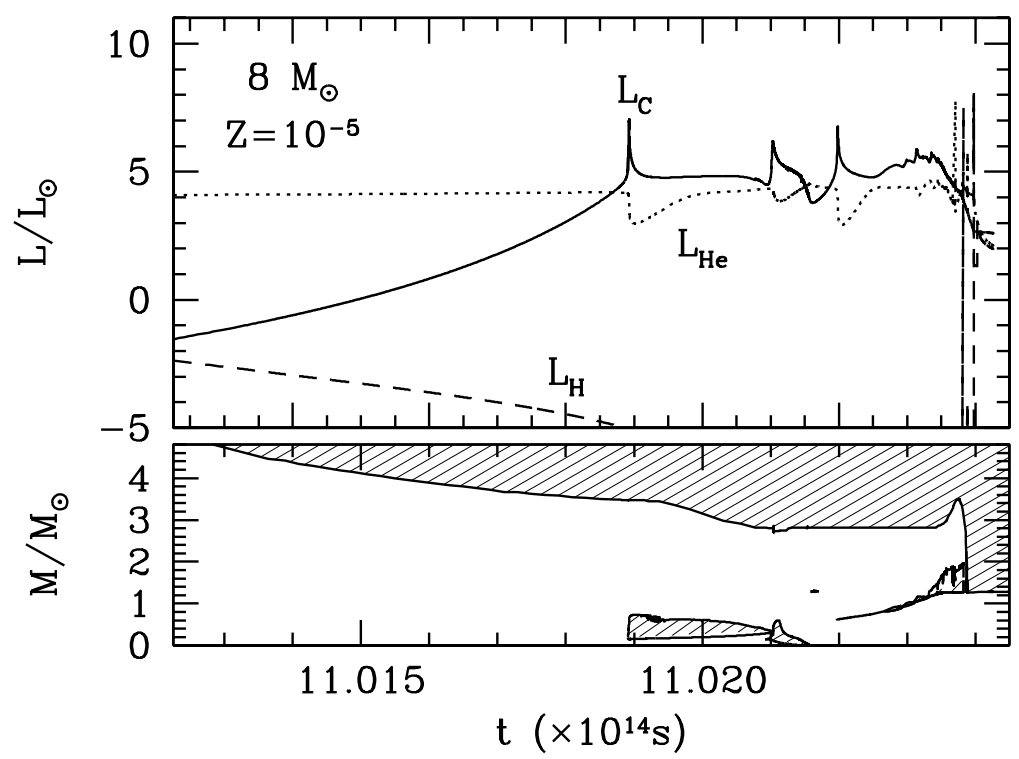

FIGURE 3. Evolution of our $8 M_{\odot}$ model during the carbon burning phase. The upper panel shows the evolution of $L_{\mathrm{H}}, L_{\mathrm{He}}$ and $L_{\mathrm{C}}$ and the lower panel shows the evolution of the convective zones.

TABLE 2. Mass of the $\mathrm{CO}$ and $\mathrm{ONe}$ cores computed with EVOLVE and no overshooting.

\begin{tabular}{ccc}
\hline$M_{\mathrm{ZAMS}} / M_{\odot}$ & $M_{\mathrm{ONe}} / M_{\odot}$ & $M_{\mathrm{CO}} / M_{\odot}$ \\
\hline 5.0 & - & 0.91 \\
6.0 & - & 0.97 \\
7.0 & 1.02 & 1.05 \\
8.0 & 1.21 & 1.22 \\
9.0 & 1.39 & 1.40 \\
\hline
\end{tabular}

resumes its advance inwards and is able to mix protons into the high temperature and density regions of the stellar interior. This causes a fast ignition of the protons, the occurrence of a hydrogen flash and the extension inwards of convection that allows the mixing of material processed during helium and carbon burning with the stellar envelope. This process was already observed and described in Ref. [17] and was later confirmed in Ref. [18], and has been referred to as dredge-out.

\section{TP-AGB EVOLUTION AND THE EFFECTS OF VARYING $f$}

Table 2 shows the sizes of the degenerate cores of the models computed with EVOLVE after the main central burning stages. The $7 M_{\odot}$ model with $Z=10^{-5}$ is already able to ignite carbon. For comparison, the minimum mass allowing carbon burning in the case 


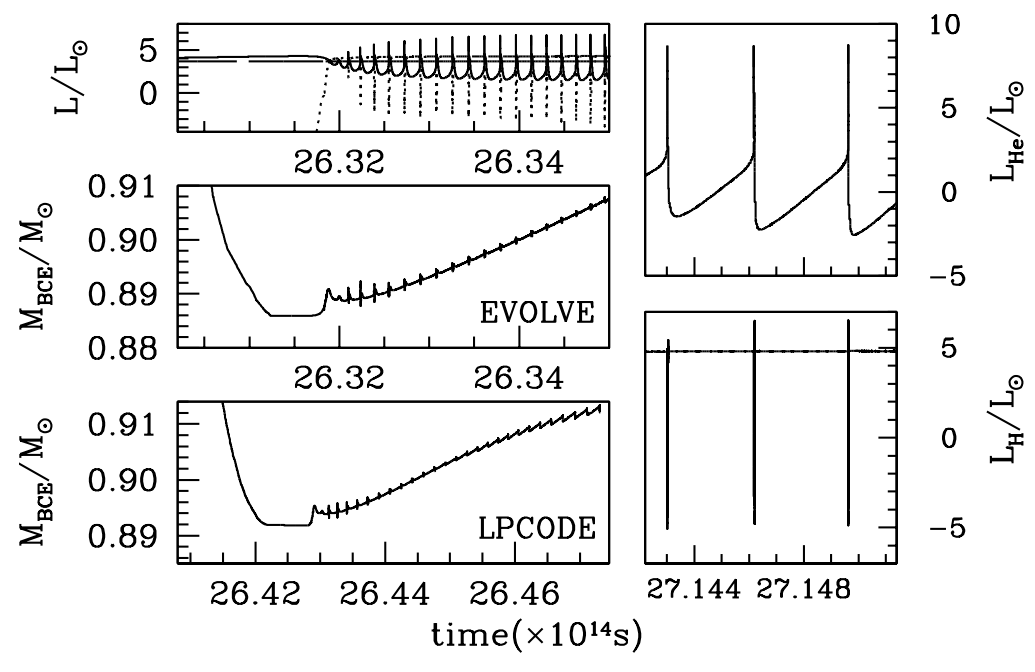

FIGURE 4. Left top panel: evolution of $L_{\mathrm{H}}$ and $L_{\mathrm{He}}$ during the early TP-AGB phase of the $5 M_{\odot}$ star computed with EVOLVE. Left middle panel: position of the base of the convective envelope (BCE) computed with EVOLVE. Left lower panel: BCE for the same model star computed with LPCODE and $f=0$. Right upper panel: evolution of $L_{\mathrm{He}}$ during the double flash events obtained with LPCODE. Right lower panel: evolution of $L_{\mathrm{H}}$ during the double flash events obtained with LPCODE.

TABLE 3. Envelope CNO abundances and total metallicity of the $5 M_{\odot}$ models computed with EVOLVE and LPCODE.

\begin{tabular}{cccccc}
\hline Code & $f$ & $X_{\text {env }}\left({ }^{12} \mathbf{C}\right)$ & $X_{\text {env }}\left({ }^{14} \mathbf{N}\right)$ & $X_{\text {env }}\left({ }^{16} \mathbf{O}\right)$ & $Z_{\text {env }}$ \\
\hline EVOLVE & - & $5.8 \times 10^{-7}$ & $3.2 \times 10^{-6}$ & $3.3 \times 10^{-6}$ & $1.10 \times 10^{-5}$ \\
LPCODE & 0.000 & $1.2 \times 10^{-6}$ & $1.4 \times 10^{-6}$ & $1.2 \times 10^{-6}$ & $1.02 \times 10^{-5}$ \\
LPCODE & 0.002 & $1.4 \times 10^{-6}$ & $1.7 \times 10^{-6}$ & $3.9 \times 10^{-6}$ & $1.06 \times 10^{-5}$ \\
LPCODE & 0.004 & $0.6 \times 10^{-6}$ & $1.5 \times 10^{-6}$ & $0.8 \times 10^{-6}$ & $1.83 \times 10^{-5}$ \\
LPCODE & 0.008 & $3.2 \times 10^{-6}$ & $3.0 \times 10^{-6}$ & $3.2 \times 10^{-6}$ & $1.32 \times 10^{-5}$ \\
LPCODE & 0.016 & $5.8 \times 10^{-5}$ & $1.1 \times 10^{-5}$ & $1.5 \times 10^{-5}$ & $1.10 \times 10^{-5}$ \\
\hline
\end{tabular}

of primordial stars is $7.8 M_{\odot}$.

Once the main central burning stages finish, the hydrogen burning shell (HBS) switches on again and the TP-AGB begins - see figure 4. On the left panels of this figure we compare the advance and retreat of the convective envelope in the evolutionary sequences calculated with EVOLVE and LPCODE. The agreement is quite close, but the importance of mixing induced by the third dredge-up (TDU) is much larger in the tracks computed with LPCODE. By this time the stellar envelope material has already been mixed with the products synthesized in the interior of the star.

We have followed the first thermal pulses of the $5 M_{\odot}$ model star using both codes and different values of $f$. Table 3 shows the chemical composition of the envelope for this case during the TP-AGB phase. The models computed with LPCODE show larger C/N abundance ratios in the envelope. It must be stressed, however, that we have not followed the same number of pulses for each value of $f$, and hence the direct comparison of these 
TABLE 4. Characteristic times (in years) needed for the cores to reach the Chandrasekhar mass and to remove the envelope using the mass-loss prescription of Reimers $\left(t_{\mathrm{env}}^{\mathrm{R}}\right)$ and that of Blöcker $\left(t_{\mathrm{env}}^{\mathrm{B}}\right)$.

\begin{tabular}{cccc}
\hline$M_{\text {ZAMS }} / M_{\odot}$ & $t_{\text {Ch }}(\mathbf{y r})$ & $t_{\text {env }}^{\mathrm{R}}(\mathbf{y r})$ & $t_{\text {env }}^{\mathrm{B}}(\mathbf{y r})$ \\
\hline 5.0 & $1.6 \times 10^{6}$ & $5.6 \times 10^{8}$ & $7.9 \times 10^{6}$ \\
6.0 & $1.5 \times 10^{6}$ & $5.2 \times 10^{8}$ & $6.5 \times 10^{6}$ \\
7.0 & $1.3 \times 10^{6}$ & $1.9 \times 10^{8}$ & $3.1 \times 10^{6}$ \\
7.5 & $9.8 \times 10^{5}$ & $1.2 \times 10^{8}$ & $2.0 \times 10^{6}$ \\
8.0 & $7.8 \times 10^{5}$ & $5.3 \times 10^{7}$ & $1.1 \times 10^{6}$ \\
8.5 & $5.4 \times 10^{5}$ & $6.0 \times 10^{7}$ & $1.4 \times 10^{6}$ \\
\hline
\end{tabular}

results can be somewhat misleading.

As to the characteristics of the TP-AGB phase, we have found that for the model computed with EVOLVE, after 15 pulses the effects of the TDU or hot bottom burning are negligible and that the envelope abundances remain constant. For the models computed with LPCODE, we have found that even for the $f=0$ case TDU and hot bottom burning play an important role. After the first 20 pulses the metallicity of the envelope changes from $Z_{\text {env }}=1.00 \times 10^{-5}$ - the second dredge-up was almost negligible for this model - to $Z_{\text {env }}=2.33 \times 10^{-4}$. Another salient feature in the LPCODE sequences is the existence of double flashes, in which protons are ingested into the He-burning shell, thus producing a proton flash that quenches He shell burning. This can be seen on the right panels of figure 4. Similar flashes have been found in Refs. [3] and [4], but a firm observational test currently seems beyond of reach.

\section{SUMMARY AND DISCUSSION}

We have used two different evolutionary codes to compute the evolution of intermediatemass extremely metal-poor stars. The two codes yield very different envelope enrichments during the TP-AGB and, therefore, point to very different possibilities for the final fates of the stars we are considering. According to the results obtained using EVOLVE, the envelope enrichment due to the TDU is almost negligible and, therefore, one can not assure that stellar winds will be powerful enough to remove the envelope before the degenerate core reaches the Chandrasekhar mass. As it was done in Ref. [11], an estimate of the final fate of these stars can be done. Using the prescription for stellar winds of Reimers [19] or that of Blöcker [20] and the sizes of the envelopes obtained from our sequences, we can estimate the time required by the stars to lose their envelopes $\left(t_{\text {env }}\right)$. Using the rates of core growth and the initial size of the cores, we can also estimate the time required to reach the Chandrasekhar mass $\left(t_{\mathrm{Ch}}\right)$. These times are shown in table 4. Even the values for $t_{\mathrm{env}}$ obtained with the prescription of Blöcker are larger than those for $t_{\mathrm{Ch}}$. Therefore one can not discard that these stars might end their lives as SNeI1/2.

We have assumed that for the models computed using the evolutionary code EVOLVE the envelope abundances are almost constant along the TP-AGB phase. But, as already shown, this is not the case for the models computed with LPCODE. If, instead, this 
assumption is dropped, as it stems from the calculations performed with LPCODE, the fast increase in the metal content of the envelope seems enough to support stellar winds similar to those of solar metallicity stars. Therefore, they will allow a standard evolution for the $5 M_{\odot}$ star and, therefore, it is foreseen that this model would lose its envelope and evolve to the white dwarf cooling track.

The problem of the evolution and fate of extremely low metallicity stars remains open and appears strongly dependent on the treatment of mixing in the model stars. The developement of thermonuclear flashes that alter the mixing of isotopes in ways that do not occur in solar metallicity stars and the new observations make the subject of the evolution of extremely low metallicity stars increasingly interesting and challenging.

\section{ACKNOWLEDGMENTS}

Part of this work was supported by the MCYT grant AYA05-08013-C03-01, by the European Union FEDER funds and by the AGAUR.

\section{REFERENCES}

1. Christlieb, N., Bessell, M.S., Beers, T.C., Gustafsson, B., Korn, A., Barklem, P.S., Karlsson, T., Mizuno-Wiedner, M., \& Rossi, S., 2002, Nature, 419, 904

2. Frebel, A., Aoki, W., Christlieb, N., Ando, H., Asplund, M., Barklem, P.S., Beers, T.C., Eriksson, K., Fechner, C., Fujimoto, M.Y., Honda, S., Kajino, T., Minezaki, T., Nomoto, K., Norris, J.E., Ryan, S.G., Takada-Hidai, M., Tsangarides, S., \& Yoshii, Y., 2005, Nature, 434, 871

3. Campbell, S. \& Lattanzio, J., 2007, ArXiV 0709.4567C

4. Woodward, P., Herwig, F., Porter, D., Fuchs, T., Nowatzki, A., \& Pignatari, M., 2008, in "First stars III”, AIP Conf Ser., Ed.: T. Abel, A. Heger and B. O'Shea, http: / / arXiv: 0711.2091

5. Arnett, D., this volume

6. Poelarends, A., 2006, Ph.D. Thesis

7. Gil-Pons, P., Gutiérrez, J. L., \& García-Berro, E., 2007, A\&A, 464, 667

8. Lau, H., Stancliffe, R., \& Tout, C., 2007, MNRAS, 378, 563

9. Arnett, D., 1968, Ap\&SS, 5, 180

10. García-Berro, E., \& Iben, I. Jr., 1994, ApJ, 434, 306

11. Gil-Pons, P., et al., 2005, A\&A, 433, 1037

12. Schröder, Pols, O., \& Eggleton, P., 1997, MNRAS, 285, 696

13. Eldridge, J., \& Tout, C., 2004, MNRAS, 353, 87

14. Althaus, L., et al., 2003, A\&A, 404, 593

15. Herwig, F., 2000, A\&A, 360, 952

16. Herwig, F., 2000, ApJ, 605, 425

17. Ritossa, C., García-Berro, E., \& Iben, I. Jr., 1999, ApJ 515, 381

18. Siess, L., 2007, A\&A, 476, 893

19. Reimers, D., 1975, Mem. Soc. Roy. Sci. Liege, 8, 369

20. Blöcker, T., 1995, A\&A, 297, 727 